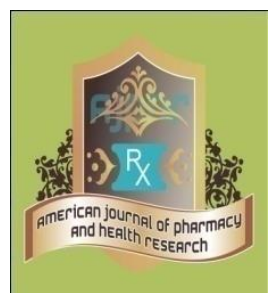

Review Article AMERICAN JOURNAL OF PHARMACY AND HEALTH RESEARCH

www.ajphr.com

2021, Volume 9, Issue 12

ISSN: 2321-3647(online)

\title{
Theoretical Models For Photocatalysis Process
}

\author{
K.Malleswari*, D.Rama Bramha Reddy ${ }^{1}$, SK Abdul Raheman ${ }^{1}$, Y.Susmitha ${ }^{1}$, \\ Sk.Ramjanbi ${ }^{1}$. \\ Nalanda Institute of Pharmaceutical Sciences, Kantepudi (VI), Sattenapalli (M), Guntur(DT)
}

\begin{abstract}
Photocatalysis represents a unique class of chemical transformations. It utilizes the energy delivered by light and drives reactions that are difficult, sometimes even impossible, to carry out in dark. When used for thermodynamically uphill reactions such as photosynthesis, photocatalysis promises a sustainable solution to large scale solar energy storage. Despite the longstanding interest in this process and research efforts, existing photocatalysis demonstrations are limited to academic laboratory settings. Chief among the reasons for the slow progress is the lack of suitable photocatalysts materials for large scale applications. For the purpose of effective light absorption, charge separation, and charge transfer, a large number of photocatalytic materials, including conventional semiconductors and emerging photoelectronic materials such as nanoscale plasmonic metal particles, quantum dots, and 2D materials, have been studied. ${ }^{1}$
\end{abstract}

Keywords: Photocatalysis, Solar energy, Materials, Properties, Applications. 


\section{INTRODUCTION}

Regulation of charge transfer processes in chemical systems is the basis for obtaining of one or another end product. At the same time, big possibilities occur during regulation of the chemical Processes when influencing on a system by external factors. Particularly, influence by the photons of defined energy allows realization of such complex chemical transformations in the system and obtaining of such products, receive of which is impossible in other conditions. Photocatalysis has been used for a long time, theoretical fundamentals for photo-catalysis mechanisms have been also worked out long ago. Modern theory of charge transfer processes in non-regular condensed systems increases substantially capabilities of conduction of analytic calculations of kinetic parameters of the processes, particularly for photo-catalysis process. ${ }^{2}$

\section{Photocatalysis definition and mechanism:}

During photocatalysis solid, semi conductive catalysts generates active oxygen forms on its surface at radiation of light of proper wavelength. These oxygen forms are responsible for oxidation and reduction of compounds adsorbed on catalyst surface. By definition, catalysts takes part in the reaction and speeds transformation of organic compounds, but remains in unchanged form at the end of the catalytic cycle. ${ }^{3}$

The aim of the heterogenic photocatalysis with the use of semi conductive catalyst TiO2is to perform series of redox reactions on the catalyst surface. It is exclusively connected with properties of electrons located on the outer molecular orbital. When photon energy (hv) of greater than or equal to valence to the band gap energy (TR) of $\mathrm{TiO} 2$ is illuminated onto the catalyst surface (usually $3.2 \mathrm{eV}$ - anatase or $3.0 \mathrm{eV}$ - rutile), free electrons are transferred from TR to empty conductive band (CB) within few femtoseconds (1 quadrillionth - 10-15 of second). Hence, "electron gap - electron" pairs are formed $(\mathrm{e}-\mathrm{h}+)$. In case of $\mathrm{TiO} 2$ the energy gap between bands can be balanced by delivering photon energy from near-UV, what usually corresponds to the wavelength $\lambda<400 \mathrm{~nm}$. Energy consumption and next generation of "electron gap - electron" pairs are stages that initiate photolycatalysis process. Chain redox reactions which occur on the photocatalyst surface are described by equations (1)-(11).

Photoexcitation: $\mathrm{TiO} 2+\mathrm{h} v \rightarrow \mathrm{e}-+\mathrm{h}+(1)$

Charge-carrier trapping of e-: e-CB $\rightarrow$ e-TR (2)

Charge-carrier trapping of $\mathrm{h}+\mathrm{h}+\mathrm{VB} \rightarrow \mathrm{h}+\mathrm{TR}(3)$

Electron-hole recombination: $\mathrm{e}-\mathrm{TR}+\mathrm{h}+\mathrm{VB}(\mathrm{h}+\mathrm{TR}) \rightarrow \mathrm{e}-\mathrm{CB}+$ heat $(4)$

Photoexcited e-scavenging: $(\mathrm{O} 2)$ ads $+\mathrm{e}-\rightarrow \mathrm{O} 2 \cdot-(5)$ 
The e-TR and h+TR in equations (1)-(4) correspond to electrons in valence band and electron Gaps in conductive band, respectively. Those electron gaps are carriers of trapped electrons and do not recombine immediately after their excitation with photons ${ }^{2}$. In the absence of electron Scavengers, the photo-excited electron is recombined with the valence band within few Nanoseconds with simultaneous heat dispersion (equation (4)). Thus, the presence of electrons Scavengers (acceptors) eg oxygen is crucial for recombination elongation and successful Photocatalysis performance. The action of oxygen avoiding electron recombination and Enabling formation of peroxide (O2•-) free radicals is shown in equation (5). Those radicals can Again undergo protonation forming hydroperoxide radicals (HO2•) and, finally, hydrogen Peroxide, what is shown in the equations.

$\mathrm{O} 2 \bullet-+\mathrm{H}+\rightarrow \mathrm{HO} 2 \bullet(6)$

$\mathrm{O} 2 \bullet-+\mathrm{O} 2 \bullet-\rightarrow \mathrm{H} 2 \mathrm{O} 2+\mathrm{O} 2(7)$

The HO2 - Radicals also act as electrons scavenger and thus, according to their coexistence, the Recombination time of $\mathrm{h}+\mathrm{TR}$ in the entire photocatalysis reaction, can be doubled. Interaction of Electron gaps with water particles and hydroxyl ions results in formation of highly reactive Hydroxyl radicals:

Oxidation of water: $\mathrm{H} 2 \mathrm{O}+\mathrm{h}+\rightarrow \mathrm{OH} \bullet+\mathrm{H}+(8)$

Oxidation hydroxyl ions: $\mathrm{OH}-+\mathrm{h}+\rightarrow \mathrm{OH} \bullet(9)$

Those radicals are bonded to the surface of hydrated metal oxide and act as a basic oxidant in Photocatalytic system.

Photo degradation caused with $\mathrm{OH} \bullet$ Radicals: $\mathrm{Re}-\mathrm{H}+\mathrm{OH} \bullet \rightarrow \mathrm{R} \bullet+\mathrm{H} 2 \mathrm{O}$ $(10) \mathrm{R}+\mathrm{h}+\rightarrow \mathrm{R}+\bullet \rightarrow$ Intermediate(s) - final degradation products (11)

\section{TYPES OF PHOTOCATALYSIS:}

HOMOGENEOUS PHOTOCATALYSIS:

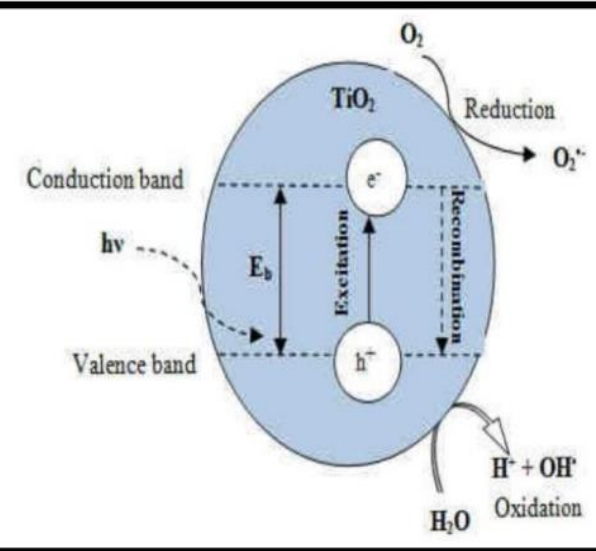

Figure 1: Photo induced formation of electron-hole pair in semiconductor $\mathrm{TiO}_{2}$ particle 
In homogeneous photocatalysis, the reactants and the photocatalysts exist in the same phase. The most commonly used homogeneous photocatalysts include ozone and photo-Fenton systems $(\mathrm{Fe}+$ and $\mathrm{Fe}+/ \mathrm{H} 2 \mathrm{O} 2)$. The reactive species is the $\bullet \mathrm{OH}$ which is used for different purposes. The mechanism of hydroxyl radical production by ozone can follow two paths.

$\mathrm{O} 3+\mathrm{h} v \rightarrow \mathrm{O} 2+\mathrm{O}(1 \mathrm{D})$

$\mathrm{O}(1 \mathrm{D})+\mathrm{H} 2 \mathrm{O} \rightarrow \cdot \mathrm{OH}+\cdot \mathrm{OH}$

$\mathrm{O}(1 \mathrm{D})+\mathrm{H} 2 \mathrm{O} \rightarrow \mathrm{H} 2 \mathrm{O} 2$

$\mathrm{H} 2 \mathrm{O} 2+\mathrm{h} v \rightarrow \cdot \mathrm{OH}+\cdot \mathrm{OH}$

The efficiency of Fenton type processes is influenced by several operating parameters like concentration of hydrogen peroxide, $\mathrm{pH}$ and intensity of UV. The main advantage of this process is the ability of using sunlight with light sensitivity up to $450 \mathrm{~nm}$, thus avoiding the high costs of UV lamps and electrical energy. These reactions have been proven more efficient than the other photocatalysis but the disadvantages of the process are the low $\mathrm{pH}$ values which are required, since iron precipitates at higher $\mathrm{pH}$ values and the fact that iron has to be removed after treatment. $^{(4)}$

\section{Heterogeneous photocatalysis:}

Heterogeneous catalysis has the catalyst in a different phase from the reactants. Heterogeneous photocatalysis is a discipline which includes a large variety of reactions: mild or total oxidations, dehydrogenation, hydrogen transfer, 1802-1602 and deuterium-alkane isotopic exchange, metal deposition, water detoxification, gaseous pollutant removal, etc.

Most common heterogeneous photocatalysts are transition metal oxides and semiconductors, which have unique characteristics. Unlike the metals which have a continuum of electronic states, semiconductors possess a void energy region where no energy levels are available to promote recombination of an electron and hole produced by photo activation in the solid ${ }^{5}$

\section{Oxidative reactions due to photocatalytic effect:}

$\mathrm{H}++\mathrm{H} 2 \mathrm{O} \rightarrow \mathrm{H}++\cdot \mathrm{OH}$

$2 \mathrm{~h}++2 \mathrm{H} 2 \mathrm{O} \rightarrow 2 \mathrm{H}++\mathrm{H} 2 \mathrm{O} 2$

$\mathrm{H} 2 \mathrm{O} 2 \rightarrow 2 \cdot \mathrm{OH}$

Reductive reactions due to photocatalytic effect:

$\mathrm{E}-+\mathrm{O} 2 \rightarrow \cdot \mathrm{O}_{2}-$

$\cdot \mathrm{O} 2-+\mathrm{H} 2 \mathrm{O}+\mathrm{H}+\rightarrow \mathrm{H} 2 \mathrm{O} 2+\mathrm{O} 2$

$\mathrm{H} 2 \mathrm{O} 2 \rightarrow 2 \cdot \mathrm{OH}$

\section{Therotical models:}




\section{Kinetic of photocatalytic reactions:}

Understanding and modeling kinetics is an essential part of the optimization and implementation of chemical reactions. In the case of photocatalytic reactions this is mostly done onedimensionally, i.e., only considering the effect of one parameter at the same time. However, as discussed in this study, many of the relevant reaction parameters have mutual interdependencies that call for a holistic multi-dimensional approach to accurately model and understand their influence. Such an approach is described herein, and all the relevant equations given so that researchers can readily implement it to analyze and model their reactions ${ }^{6}$

\section{Degradation and dissection of water pollutants:}

Industrial wastewaters contain a variety of toxic and carcinogenic Compounds (e.g. pharmaceuticals, pesticides, and surfactants), which Pose a threat to the environment if released without treatment; as de-Scribed in several recent review articles. Most of these com-Pounds are persistent and pass through conventional biological treatment systems without significant changes in their chemistry or Concentration. Therefore, advanced water treatment Technologies are essential before industrial wastewater containing such Compounds can be discharged into the sewer network or water bodies. Advanced oxidation processes (AOPs) are recognized as being effective For the degradation of a variety of bio-recalcitrant compounds including Phenols, pesticides, pharmaceuticals, dyes, and petrochemicals. In AOPs, reactive oxygen species (ROS) oxidize Organic molecules and break them down into degradation products.These reactions can be enhanced by light and/or catalysts. Among AOPs, heterogeneous photocatalysts employ solid semicon-Ductor catalysts illuminated at a wavelength of light exceeding the Bandgap to degrade organic pollutants. ${ }^{7}$

\section{Photocatalytic reactor modelling:}

Various types of reactors have been used in the photocatalytic water treatment, including the annular slurry photoreactor, cascade photoreactor, down flow contactor reactor, etc. The disparity between these two main configurations is that the first one requires an additional downstream separation unit for the recovery of photocatalyst particles while the latter permits a continuous operation. The most important factors in configuring a photocatalytic reactor are the total irradiated surface area of catalyst per unit volume and light distribution within the reactor. ${ }^{8}$ Slurry-type photocatalytic reactor usually performs a high total surface area of photocatalyst per unit volume, while the fixed-bed configuration is often associated with mass transfer limitation over the immobilized layer of photocatalysts. The light photon distribution through either direct or diffuse paths within the reactors needs to be decided. Direct photon utilization means that the 
photocatalysts are directly activated with light photon, rather with the assistance of various parabolic light deflectors to transfer the photons. To achieve uniformity in photon flux distribution within the reactor, a correct position of light source is essential to ensure maximal and symmetrical light transmission and distribution. The use of photo reactors with assisted parabolic light deflectors nowadays have become unfavorable, owing to the need of special configuration and high operating costs. This type of reactor needs to be specifically designed to ensure the maximal illuminated reactor volume with minimal pressure requirement for good catalyst mixing and dispersion. Until recently, the slurry photocatalytic reactor was still the preferred configuration owing to its high total surface area of photocatalyst per unit volume and ease of photocatalysts reactivation.

\section{Photocatalysis membrane reactor:}

The hybrid systems comprised of photocatalytic reactor and membrane process are known as Membrane photocatalytic reactors. The membrane module can be placed outside or Inside the photocatalytic reactor. ${ }^{9}$

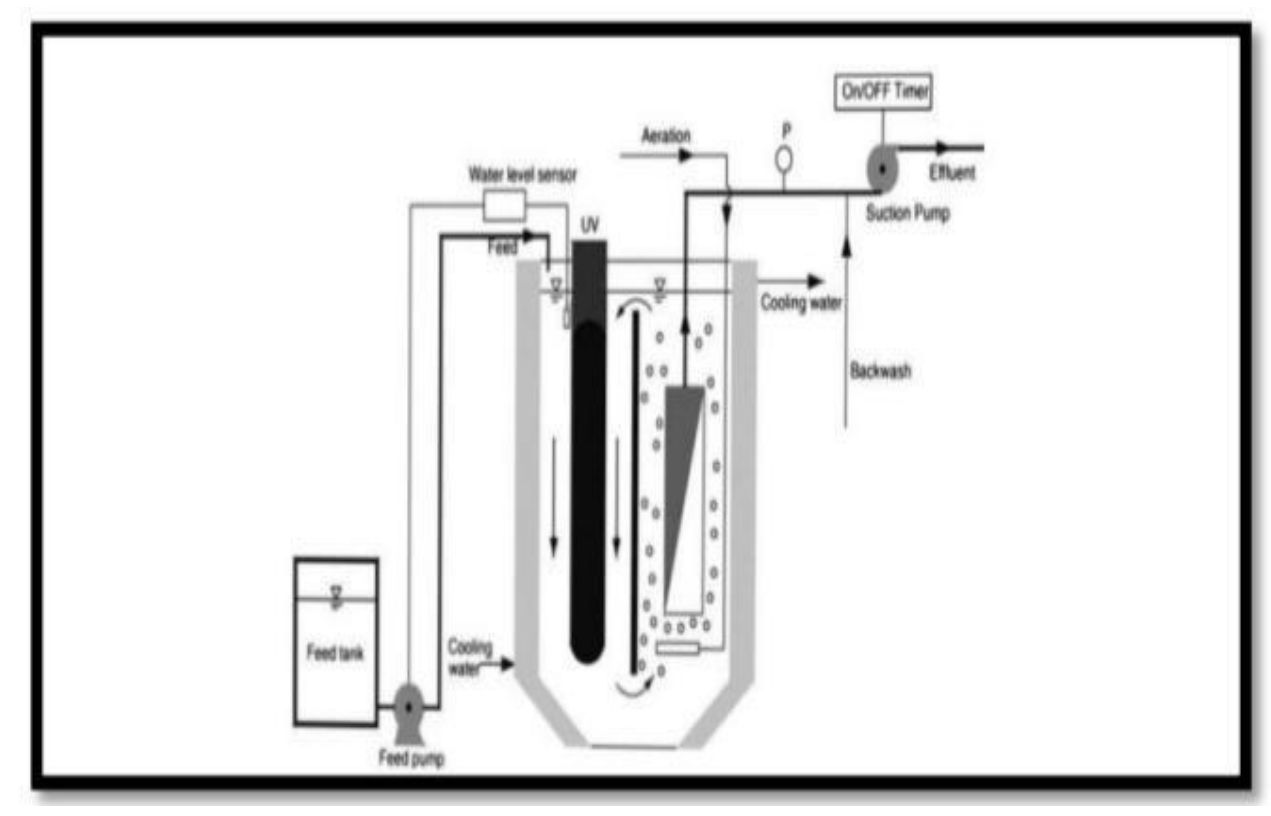

Figure:2 Photo catalytic membrane reactor

In case when the membrane module is placed outside the reactor three different configurations Can be distinguish i.e the source of light is located over the feed tank, over the membrane module Or over the additional tank (photoreactor) which is situated between feed tank and membrane Module. In some cases the source of light can be placed over both, membrane module and Feed tank (or inside feed tank in case of immersed UV lamps). Immersed membrane reactor Consists of reaction zone, in which $\mathrm{TiO} 2$ suspension radiated with UV rays is separated from Membrane module by moveable baffle. 
Generally, such reactors can be designed with irradiation of membrane module or suspended Catalyst feed tank. In suspended reactors the membrane acts as physical barrier for both, Photocatalyst particles and/or organic molecules and reaction by-products. In the first Configuration the photocatalyst can be either suspended in water phase or immobilized on the Membrane. In membrane reactor with immobilized catalyst membrane module is used as a Support for photocatalyst particles (photocatalytic membranes) and acts as a barrier for organic Molecules or microorganisms present in the treated mixture.

\section{INFLUENCE OF PROCESS PARAMETERS ON PHOTOCATALYSIS}

\section{Effectiveness:}

Photocatalytic oxidation effectiveness is depended on series of operational parameters, which impact on the process is discussed below.

\section{Catalyst dose:}

The increase of catalyst dose results in increase of active surface available for Adsorption and degradation processes. On the other hand, the turbidity of the solution Increases, thus the penetration of stream by photons is limited. Moreover, the surface decrease can be also observed as at high solid concentration $\mathrm{TiO} 2$ particles form agglomerates. Hence, the initial rate of photoreaction and its effectiveness are directly proportional to catalyst mass. However, when the critical dose is exceeded, the reaction rate is independent from it and remains constant or even decreases with the catalyst dose increase. Thus, the proper estimation of optimal catalyst dose must be done for a given application (i.e reactor configuration, source of radiation, contaminants concentration, etc).

\section{PH:}

The influence of $\mathrm{pH}$ on photocatalytic degradation of organic compounds in water is mainly related to the rate of ionization of $\mathrm{TiO} 2$ surface, position of valence and conductive bands of photocatalyst, the degree of $\mathrm{TiO} 2$ agglomeration and formation of hydroxyl radicals. The isoelectric point of $\mathrm{TiO} 2$ (zero charge point - ZCP) changes within $\mathrm{pH}$ range 6-7 depending on the catalyst applied. For commercial TiO2 (eg Degussa P25, ZCP is obtained at $\mathrm{pH}=6.8$ ) at $\mathrm{pH}$ $<\mathrm{ZCP}(\mathrm{TiO} 2)$ the surface charge of the catalyst becomes positive and step wisely increases the electrostatic attraction of negatively charged compounds. Such a polar interaction between $\mathrm{TiO} 2$ and charged anionic organic compounds can intensify the adsorption on the $\mathrm{TiO} 2$ surface, which is activated with photons for next photocatalytic reactions. It is especially important in case of low concentration of anionic organic compounds. At $\mathrm{pH}>\mathrm{ZCP}$ (TiO2) the catalyst.

At $\mathrm{pH}<\mathrm{PZC}: \mathrm{TiOH}+\mathrm{H}+\leftrightarrow \mathrm{TiOH} 2+(12)$ 
At $\mathrm{pH}>\mathrm{PZC}: \mathrm{TiOH}+\mathrm{OH}-\leftrightarrow \mathrm{TiO}-+\mathrm{H}_{2} \mathrm{O}(13)$

\section{Temperature:}

Many studies have revealed the dependence of the effectiveness and rate of the Photocatalysis On the temperature. Generally, the process should be carried out at The temperature range of $20 \div 80^{\circ} \mathrm{C}$. At very low temperature (ie below $0^{\circ} \mathrm{C}$ ) the Activity of the catalyst decreases and The desorption of the final product from the catalyst Surface becomes the limiting step of the Reaction rate. On the other hand, at temperature Above $80^{\circ} \mathrm{C}$, the adsorption of contaminants is Ineffective and thus the photoreactionTiO2 Performance is limited. At the temperature range $20 \div 60^{\circ} \mathrm{C}$ the increase of the Photoreaction rate with the increase of the temperature is observed. The activation Of surface and the proper understanding of the reaction rate dependence on the Temperature is very important when the process is carried out under natural sunlight Conditions. Such A dependence is Described by Van't Hoff-Arrhenius equation, in which constant is linearly Proportional to $\operatorname{Exp}(-1 / \mathrm{T})$ :

$$
\ln \left(\frac{k_{1}}{k_{2}}\right)=-\frac{E_{a}}{R}\left(\frac{1}{T_{2}}-\frac{1}{T_{1}}\right)
$$

Where

$\mathrm{k} 1$ and $\mathrm{k} 2$ are the constants for temperature

$\mathrm{T} 1$ and $\mathrm{T} 2$, Ea is the energy of activation and

$\mathrm{R}$ is the universal gas constant.

The vitality of microorganisms with respect to the catalyst Activity depends on the incubation temperature and type and resistance to temperature change. Generally, the order of resistance of microorganisms to conventional disinfection systems is Of: non-spore forming bacteria < viruses $<$ spore forming bacteria < parasites < protozoa (oocytes). ${ }^{10}$

\section{Dissolved oxygen:}

The concentration of the dissolved oxygen in the photocatalytic system with $\mathrm{TiO} 2$ is very important as it provides the efficient electron scavenging in the conductive band and prevents. Their recombination. The presence of the dissolved oxygen also influences the formation of oxygen-containing radicals other than hydroxyl ones and guarantees the stability of semi radicals, mineralization and photocatalytic reaction itself. The dissolved oxygen also allows holding $\mathrm{TiO} 2$ particles in the suspension. However, the operation of photoreactor equipped with the pure oxygen supply is very expensive. Thus, it can be replaced with air as any significant differences in the process performance have been observed. 


\section{Initial concentration of reagents:}

In general, the rate of organic contaminants degradation increases with the increase of Substrates initial concentration. However, this increase is observed only to a given point, Exceeding of which results in the decrease of the degradation rate. The study confirmed that at the similar process conditions the change of the initial water contaminants concentration causes the change of necessary radiation time required for total disinfection or mineralization. In respect to the photonic character of the photocatalytic reaction the excessive concentration of organic substrates causes both, the saturation of $\mathrm{TiO} 2$ surface and the reduction of Photocatalysis efficiency, what results in the inactivation of the photocatalyst. Moreover, the Time of radiation strongly depends on the chemical character of organic substrates.

\section{Feed water quality:}

In practical application photocatalysis can be used for treatment of water of various Qmeters have a great impact on the performance of advanced Photocatalytic processes with $\mathrm{TiO} 2$.

\section{Turbidity:}

Which is caused by insoluble solid particles present in the raw water, is a significant Disruptor of photocatalytic process based on $\mathrm{TiO} 2$. Suspended or dispersed particles have a huge Influence on optical water properties and further impede the transmission of UV light by strong Dispersion and adsorption of the rays. It causes changes in expected $\mathrm{TiO} 2$ charge and affects Pathway and intensity of UV light. Moreover, a significant turbidity may cause shielding effect, which decreases light transmission, protects contaminants from UV rays action and limits the Performance of both, photocatalysis and photodisinfection. Finally, the action of all those Factors causes the ineffective photocatalytic water treatment. In order to ensure the proper rate Of photocatalytic reaction the water turbidity should not exceed 5 NTU what enables the Optimal use of UV light

\section{APPLICATIONS:}

- Paper production: Large scale photocatalysis by micro-sized $\mathrm{ZnO}$ tetrapodal particles added to pilot paper production.

- Separating water into hydrogen and oxygen by photocatalytic water splitting. Usage of fossil fuels is causing a huge amount of air pollutants, such as nitrogen oxides, sulfur oxides, and carbon oxides. Using sunlight as a renewable energy source is therefore becoming increasingly interesting.

- Use of titanium dioxide in self-cleaning glass. Free radicals generated from TiO2 oxidize organic matter. 
- Disinfection of water by supported titanium dioxide photocatalysts, a form of solar water disinfection (SODIS).

- Sterilization of surgical instruments and removal of unwanted fingerprints from sensitive electrical and optical components.

\section{CONCLUSION:}

Semi conductive photocatalysis with the use of UV or sun radiation has become the ustable method for both, organic compounds mineralization and disinfection. It is very important .Considering refractive organic substances, which are not transported to different phase, but also completely mineralized. The integration of the process with other treatment techniques creates a serious alternative for other methods applied in water and wastewater treatment. In order to spread the application of photocatalytic process for water and wastewater technology number of operational improvements should be made ie the development of catalytic material of high photo effectiveness. which can be used in wider radiation range (visible light radiation), immobilization of catalyst to assure economically attractive solid liquid separation, the expansion of operational $\mathrm{pH}$ range and minimization of additional Oxidants dosing, development of new systems improving photo-mineralization and photo disinfection kinetics, effective design of photocatalytic reactors or parabolic sun collectors to increase the share of sun energy and decrease energy cost.

\section{REFERENCE:}

1. Tong, Y.; Liang, J.; Liu, H.K.; Dou, S.X. "Energy storage in Oceania": Energy Storage Mater;2019; 20(11):176-187.

2. Marsagishvili TA. Heterogeneous process of charge transfer and phototransfer With participation of dipole particles. Journal of Electro anal Chem. 1998;450:47.

3. Wang, B.; Shen, S.; Mao, S.S. Black “TiO2for solar hydrogen Conversion”:Mater.2017: 3(7);96-111.

4. Cie'sla. Homogeneous photocatalysis by transition metal complexes in the environment". Journal of Molecular Catalysis A: Chemical 224 (2004) 17-33: 23.

5. Linsebigler, Amy L.; Lu, Guangquan.; Yates, John T. (1995). "Photocatalysis on TiO2 Surfaces: Principles, Mechanisms, and Selected Results”. Chemical Reviews. 95 (3): 735-758.

6. Dillert, R., Engel, A., Große, J., Lindner, P., and Bahnemann, D. W. Light intensity dependence of the kinetics of the photocatalytic oxidation of nitrogen (ii) oxide at the surface of TiO2. Phys. Chem. Chem. Phys. 2013; 15:20876. 
7. Alalm, M.G., Samy, M., Ookawara, S., Ohno, T., 2018. Immobilization of S-TiO2 on reusable aluminum plates by polysiloxane for photocatalytic degradation of 2, 4dichlorophenol in water. Journal of water process engineering 26, 329-335.

8. Tanaka, K.; Capule, M.F.V.; Hisanaga, T. "Effect of crystallinity of TiO2 on its Photocatalytic action":Chem. Phys. Lett. 1991; 187(28);73-76.

9. Kudo, A.; Miseki, Y. "Heterogeneous photocatalyst materials for water Splitting":Chem. Soc. Rev. 2009;38(82);253-278.

10. Khan, S.U.M.; Sultana, T. "Photoresponse of n-TiO2thin film and nanowire Electrodes”:Sol. Energy Mat. Sol. Cells 2003; 76(34); 211-22.

AJPHR is

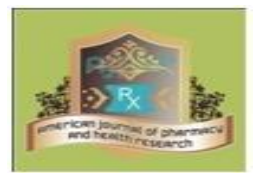

Peer-reviewed

monthly

Rapid publication

Submit your next manuscript at

editor@ajphr.com / editor.ajphr@gmail.com 